\title{
Cicatrización periodontal en pacientes con diabetes mellitus
}

\author{
Periodontal Cicatrization in Patients With Diabetes Mellitus
}

Britto Falcón Guerrero ${ }^{1 a}$, Sandra Quea Ticona ${ }^{1 b}$, Misthi Quispe Quispe ${ }^{1 b}$, Solange Nina Charca ${ }^{1 \mathrm{~b}}$, Willy Medina Condori, ${ }^{1 \mathrm{~b}}$, Niria Tito Mamani ${ }^{1 \mathrm{~b}}$, Daniela Pérez Ticona ${ }^{1 \mathrm{~b}}$

https://orcid.org/0000-0002-9585-7052

\section{Resumen}

La diabetes es una enfermedad crónica, común y compleja, que cada vez tiene más presencia dentro de la población mundial debido a los desórdenes alimenticios de las personas. Esta enfermedad también tiene un efecto adverso en los tejidos periodontales y sobre todo en el proceso de cicatrización, lo cual afecta la formación del colágeno y a los factores de crecimiento. La cicatrización es el proceso por el cual ocurre el reemplazo de tejido afectado por uno nuevo a fin de restablecer su función; por tanto, es importante que este proceso no sea alterado. En ese sentido, el objetivo de este artículo fue realizar una revisión bibliográfica actualizada en motores de búsqueda de revistas indexadas (PubMed y SciELO) buscando evidenciar la relación entre la diabetes y la cicatrización de los tejidos periodontales en pacientes diabéticos. Al término de este proceso de revisión, se concluye que la diabetes es un factor de riesgo para una adecuada cicatrización de los tejidos periodontales. Asimismo, se resalta la importancia de verificar un nivel de glucosa estable en el paciente antes de realizar un procedimiento quirúrgico, a efecto de evitar que se presenten resultados adversos en el posoperatorio.

Palabras clave: diabetes mellitus, cicatrización de heridas, periodoncia, tejido periodontal, glucosa (DeCS)

\section{Abstract}

Diabetes is a chronic, common and complex disease that has an increasing presence within the world population due to people's eating disorders. This disease also has an adverse effect on the periodontal tissues and especially on the healing process, which affects the formation of collagen and growth factors. Healing is the process by which the affected tissue is replaced by a new one in order to restore its function; therefore, it is important that this process is not disturbed. In this sense, the objective of this article was to carry out an updated bibliographic review in search engines of indexed journals (PubMed and SciELO) searching to demonstrate the relationship between diabetes and healing of periodontal tissues in diabetic patients. At the end of this review process, it is concluded that diabetes is a risk factor for adequate healing of periodontal tissues. Likewise, the importance of verifying a stable glucose level in the patient before performing a surgical procedure is highlighted, in order to avoid adverse postoperative results.

Keywords: diabetes mellitus, wound healing, periodontics, glucose periodontal tissue (DeCS)

\footnotetext{
${ }^{1}$ Facultad de Odontología Universidad Latinoamericana CIMA, Tacna Perú

${ }^{a}$ Docente, Doctor en Estomatología

${ }^{\mathrm{b}}$ Estudiante
} 


\section{Introducción}

La periodontitis y la diabetes son enfermedades crónicas y complejas con una relación bidireccional establecida. Esto es, la diabetes se asocia con una mayor prevalencia y gravedad de la periodontitis y la periodontitis se asocia con el control glucémico del paciente. ${ }^{1}$ Por tanto, la salud oral se relaciona con la diabetes; consecuentemente, es importante que los diabéticos sean conscientes de su predisposición a la enfermedad periodontal (EP), ${ }^{1}$ lo cual podría afectar el sistema de soporte del diente y su posterior pérdida. ${ }^{2}$

La cirugía periodontal facilita el acceso para realizar un correcto raspado y alisado radicular a fin de restablecer la morfología gingival y el tejido conjuntivo de las apófisis alveolares y de los dientes. ${ }^{3} \mathrm{La}$ morbilidad de la diabetes aumenta con la edad, y es más predominante en el sexo femenino. Esta afección compromete la cicatrización periodontal por la alteración de la actividad celular y menor resistencia a la infección, lo cual repercute en la respuesta a la cicatrización. ${ }^{4}$

Tomando en cuenta esos antecedentes, este estudio tiene el objetivo realizar una revisión bibliográfica actualizada para evidenciar cómo sucede el proceso de cicatrización periodontal en los pacientes con diabetes mellitus.

\section{Cicatrización de heridas}

La cicatrización es un proceso complejo que involucra a numerosos actores celulares y moleculares. En tales circunstancias, la inflamación es considerada como un iniciador de la curación, siempre que no escape de los mecanismos reguladores y se torne patológica. ${ }^{5} \mathrm{La}$ curación de las heridas es un proceso que se da en cuatro pasos: 1) hemostasia; 2) inflamación; 3) proliferación/granulación celular; y 4) remodelación/maduración. Los plazos para cada paso en el proceso son entre 1-3 días, 3-20 días, 7-40 días y 40 días a 2 años, respectivamente, con superposiciones de subprocesos. ${ }^{5}$

Después de una lesión, el subendotelio expuesto, el colágeno y el factor tisular activan la agregación plaquetaria, originando el tejido de granulación y la liberación de factores quimiotácticos (quimiocinas) con factores de crecimiento. Todo esto para formar el coágulo y obtener una hemostasia exitosa. ${ }^{6}$ Los neutrófilos son los primeros en aparecer en la lesión limpiando los desechos y las bacterias, de esta forma, proporcionan un buen ambiente para la curación de la herida. Luego, los macrófagos se acumulan y facilitan la fagocitosis de las bacterias. La hemostasia y la fase inflamatoria a menudo tardan $72 \mathrm{~h}$ en terminar. $^{7}$ En la fase proliferativa (que se da entre días y semanas) la herida presenta fibroblastos, queratinocitos y células endoteliales, originando la matriz extracelular (MEC). Esta matriz incluye proteoglicanos, ácido hialurónico, colágeno y elastina, y forma el tejido de granulación que reemplaza al coágulo. Para ese efecto, participa el factor de crecimiento transformante $b$ (TGF-b, incluidos TGF-b1, TGF-b2 y TGF-b3), la familia de interleucina (IL) y los factores de angiogénesis (factor vascular de crecimiento epidérmico). ${ }^{6,7,8}$

Posteriormente, sucede el último paso de la curación que es la fase de remodelación. Esta fase necesita un equilibrio preciso entre la apoptosis celular y la producción de nuevas células. A este respecto, la degradación gradual de la MEC, el colágeno inmaduro tipo III y la formación de colágeno maduro tipo I son críticos en esta fase. La duración de esta fase oscila entre unos meses a años. ${ }^{6,7,8}$

\section{Cicatrización periodontal}

Durante la cicatrización de un paciente sano en la MEC se libera un número significativo de proteínas de señalización celular (factores de crecimiento, quimiocinas o citocinas) y productos de la actividad celular (enzimas, moléculas de adhesión). posterior a la lesión tisular asociada con la cirugía periodontal, en una situación sin diabetes el epitelio posee la E-cadherina que es una molécula de adhesión que actúa en el mantenimiento de la integridad estructural y la función de la barrera epitelial, pero esta molécula de adhesión esta reducida en el proceso de periodontitis. ${ }^{9}$ El factor de crecimiento epitelial (EGF) juega un papel importante en la estimulación de la proliferación y diferenciación del epitelio, de los tejidos mesenquimales y la reepitelización 
de la herida cuando hay una lesión aguda. ${ }^{9} \mathrm{En}$ el tejido conectivo, el factor de crecimiento transformante beta 1 (TGF-b1), el factor de crecimiento endotelial vascular (VEGF), el factor de crecimiento de fibroblastos 2 (FGF2), la metaloproteinasa de matriz 1 (MMP-1) y las metaloproteinasas inhibidoras de tejido (TIMP-1) son proteínas de señalización celular que funcionan orquestando y estimulando la angiogénesis, la formación de tejido de granulación, la regeneración y remodelación del tejido conectivo. En tales circunstancias, la MMP-1, perteneciente a la familia de las metaloproteinasas de la matriz -MMP, desempeña un papel importante en el mantenimiento de la matriz extracelular, en la migración de queratinocitos, en la reepitelización y en la diferenciación osteoblástica. ${ }^{9,10}$

La MEC proporciona un andamio que promueve la repoblación de fibroblastos, vasos sanguíneos y epitelio de los tejidos adyacentes, los cuales a su vez estimulan la adhesión, diferenciación y proliferación celular; también, facilitan la angiogénesis aportando vascularización. Posteriormente, las células epiteliales migran rápidamente cubriendo el coágulo de fibrina donde se forma una barrera epitelial de protección que es similar al epitelio original. ${ }^{11}$

La herida periodontal ocasiona una situación compleja, ya que los márgenes de la herida no son dos superficies vasculares opuestas, sino una relación entre una superficie rígida avascular y el tejido conectivo del colgajo. En esa secuencia, se forma un primer evento de curación en la interfaz raíz y colgajo, es decir, la absorción y adhesión de proteínas plasmáticas en la superficie de la raíz. ${ }^{12}$ Seguidamente, a los pocos minutos se forma un coágulo de fibrina unido a la superficie de la raíz; luego, las células inflamatorias (neutrófilos y monocitos) se acumulan en la superficie de la raíz en una cuestión de horas. El proceso de curación domina aproximadamente al tercer día la fase tardía de la inflamación. ${ }^{12} \mathrm{Y}$, a los siete días, se observa un accesorio de tejido conectivo en la superficie de la raíz con áreas del coágulo de fibrina en diversas etapas de maduración. ${ }^{12}$

Además, el cierre por primera intención es un factor crucial para el éxito y mantenimiento de la estabilidad de la herida en las primeras semanas posquirúrgicas. ${ }^{13}$ Esto sugiere que la estabilización de los colgajos solo sea posible por la sutura durante la fase temprana, siendo importante la elección del material de sutura para los procedimientos periodontales regenerativos y los cuidados post operatorios, los mismos que deben destinarse a proteger el sitio quirúrgico de la infección. ${ }^{12}$ Todo esto con el fin de regenerar del tejido nativo, reconstituyendo y restaurando su arquitectura y función. ${ }^{14}$

También, se debe considerar los factores que pueden interferir en el proceso normal de cicatrización, tales como: a) déficit proteico y vitamínico, que obstaculiza la síntesis de colágeno y fibroblastos; b) radiación terapéutica, que altera la vascularidad reduciendo el potencial óseo para la reparación; c) vejez, que reduce la respuesta del organismo y su capacidad regeneradora; d) trastornos metabólicos (diabetes, hipercalcemia), que ocasiona una cicatrización tisular deficiente y una menor defensa ante la infección; e) trastornos medicamentosos (antimetabólicos, inmunosupresores) y hormonales. ${ }^{15}$

También se debe considerar que Pellegrini et al. ${ }^{16}$ indicaron que la expresión local de la matriz de metaloproteinasa-1 y la proteína morfogenética ósea-7 se asocian con el éxito clínico de la cirugía periodontal. Igualmente, Chen et al. ${ }^{17}$ destacan la importancia que tiene el diseño del colgajo para no interferir en la cicatrización periodontal.

\section{Diabetes}

La diabetes es un grupo de trastornos metabólicos caracterizados por hiperglucemia y causa complicaciones macro y microvasculares, afectando a los órganos del cuerpo, presentándose como diabetes tipo 1, tipo 2 y la diabetes gestacional. ${ }^{1,18}$

La diabetes mellitus tipo 1 (DM1), se produce por la deficiencia de insulina y se diagnostica a edad temprana. Es de inicio rápido, raramente diagnosticada y es ocasionada por la destrucción autoinmune de las células $\beta$ del páncreas. ${ }^{19} \mathrm{La}$ diabetes mellitus tipo 2 (DM2), se presenta a mediana edad y puede permanecer sin diagnostico 
porque la hiperglucemia aparece gradualmente y a menudo no manifiesta sintomas. ${ }^{20}$ Este tipo surge de las interacciones entre factores medio ambientales que originan la resistencia a la insulina y las manifestaciones clínicas de la enfermedad; de modo tal, que se presenta alteración en la producción de insulina, debido a que no se observa destrucción autoinmune de las células $\beta .^{20}$

La diabetes gestacional ocurre en gestantes sin antecedentes de diabetes que desarrollan hiperglucemia durante el embarazo. ${ }^{1,18}$ Presenta reducción de secreción y resistencia a la insulina, ${ }^{1,18}$ y suele mejorar después del embarazo. ${ }^{1,18}$

\section{Cicatrización periodontal en pacientes diabéticos}

La principal complicación de una herida quirúrgica, es la morbilidad postoperatoria que no se resuelve hasta que suceda la epitelización de la herida, lo cual demora de 2 a 4 semanas. ${ }^{21}$ Sin embargo, se ha demostrado una correlación positiva entre la DM2 y la EP, ${ }^{19}$ ocasionando deterioro de la cicatrización y el desarrollo de lesiones perirradiculares; ${ }^{20}$ además, esto afecta negativamente los resultados del tratamiento periodontal no quirúrgico y quirúrgico. ${ }^{22}$

En la diabetes, la cicatrización se torna defectuosa por el deterioro de la activación y respuesta de los neutrófilos, la migración y proliferación de fibroblastos, y la angiogénesis; ${ }^{23,24}$ debido a que los diabéticos tienen niveles elevados de productos finales de glicación avanzada en los tejidos gingivales que interfieren con las interacciones matriz-célula, ${ }^{23,24}$ al alterar la reticulación de la MEC y perjudicar la cicatrización de heridas. ${ }^{23,24}$

Ghaisas et al. ${ }^{25}$ mencionan que el deterioro de la angiogénesis y falla en las funciones de la matriz de metaloproteinasas (MMP), queratinocitos y fibroblastos; afecta la curación y hace al paciente más propenso a infecciones y úlceras. Kido et al. ${ }^{19}$ confirmaron que debido a la deficiencia de insulina se afecta el proceso de curación a través de la resistencia a la insulina causada por la hiperglucemia. Demostrando, que el estrés oxidativo inducido por la glucosa alta puede causar resistencia a la insulina de los fibroblastos gingivales originando una proliferación celular anormal y la cicatrización tardía. Del mismo modo, Li et al. ${ }^{26}$ mencionan que una alta concentración de glucosa puede comprometer la curación y la regeneración periodontal en pacientes diabéticos.

Shirakata et al. ${ }^{27}$ hallaron que en procesos regenerativos con matriz derivada del esmalte, se presentó inflamación excesiva debido a la colonización bacteriana, anquilosis y fracturas óseas, en animales diabéticos.

Chang et al. ${ }^{21}$ mencionan que los productos finales de la glicación avanzada (AGE) se acumulan en los tejidos de animales con diabetes, así como en los tejidos periodontales que están experimentando inflamación progresiva. Lo que interfiere con las interacciones matriz-célula, al alterar la reticulación de la matriz extracelular y perjudica así la cicatrización de las heridas. Encontrando que los agentes anti-AGE (aminoguanidina y la sintasa nítrica oxidasa inducible) parecen facilitar la cicatrización de heridas al reducir la inflamación asociada a la AGE y promover el proceso de cicatrización.

Yaman et al. ${ }^{22}$ investigaron la regulación de las citocinas en las heridas orales diabéticas que podrían estar relacionadas con la disminución de la angiogénesis, el reclutamiento disminuido de las células del músculo liso y la cicatrización tardía de las heridas, en DM2; encontrando que histológicamente, la migración del tejido epitelial para el cierre de la herida se retrasó.

Un punto importante a tomar en cuenta, en base a los antecedentes revisados, es que todos los estudios mencionan que los participantes en grupos control, donde el nivel de glucosa está controlado, no sufren alteraciones, retrasos o cuadros infecciosos en las zonas de las heridas.

\section{Conclusiones}

La diabetes es un factor de riesgo que interfiere con la cicatrización de los tejidos periodontales. Por tal motivo, el paciente debe tener controlada su glucosa previa a una cirugía periodontal, a manera de evitar complicaciones durante el procedimiento quirúrgico. 


\section{Referencia}

1. Casanova L, Hughes FJ, Preshaw PM. Diabetes and periodontal disease: a two-way relationship. $\mathrm{Br}$ Dent $\mathrm{J}$. Oct 2014; $217(8): 433-437$. Doi: 10.1038/sj.bdj.2014.907.

2. Martínez GD, Pacheco PYT. Regeneración tisular guiada en paciente diabético: reporte de caso. Correo Científico Médico. 2017; 21(4):1201-1210. Disponible en: https://www.medigraphic.com/cgi$\mathrm{bin} / \mathrm{new} / \mathrm{resumen} . \mathrm{cgi}$ ?IDARTICULO=7 9787

3. Huayhua K, Huayhua R. Eficacia del clorelase y del madecassol en la cicatrización gingival en pacientes sometidos a gingivectomía. Evid. Odontol. Clin. 2015; 1(1):27-30. DOI: http://dx.doi.org/10.35306/eoc.v1i1.117

4. Rodríguez RV, Rodríguez CJD, Rodríguez MaFA, Moreno DO. Relación Diabetes Mellitus y Salud Periodontal Consultorio 39. Rosa La Bayamesa. Bayamo. Mul Med. 2019; 23(1):59-73. Disponible en: https://www.medigraphic.com/cgibin/new/resumen.cgi?IDARTICULO=8 6780 .

5. Garraud O, Hozzein WN, Badr G. Wound healing: time to look for intelligent, 'natural' immunological approaches?. BMC Immunol. 21 Jun 2017;18 (Suppl 1):23. Doi: 10.1186/s12865-017-0207-y. PMID: 28681702; PMCID: PMC5499069.

6. Lindley LE, Stojadinovic O, Pastar I, Tomic-Canic M. Biology and biomarkers for wound healing. Plast Reconstr Surg. Sept 2016;138 (Suppl. 3): 18S-28S. D 0

10.1097/PRS.0000000000002682. P MID： 27556760 ; PM C ID: PMC4998971.

7. Plikus MV, Guerrero-Juarez CF, Ito M, Li YR, Dedhia PH, Zheng Y, Shao M, Gay DL, Ramos R, Hsi TC, Oh JW, Wang X, Ramirez A, Konopelski SE, Elzein A, Wang A, Supapannachart RJ, Lee HL, Lim CH, Nace A, Guo A, Treffeisen E, Andl T, Ramirez RN, Murad R, Offermanns S, Metzger D, Chambon P,
Widgerow AD, Tuan TL, Mortazavi A, Gupta RK, Hamilton BA, Millar SE, Seale P, Pear WS, Lazar MA, Cotsarelis $G$. Regeneration of fat cells from myofibroblasts during wound healing. Science. 17 Feb 2017; 355(6326):748752. Doi: $10.1126 /$ science.aai8792

8. Wang $\mathrm{PH}$, Huang BS, Horng $\mathrm{HC}$, Yeh CC, Chen YJ. Wound healing. J Chin Med Assoc. Feb 2018;81(2):94-101. Doi: 10.1016/j.jcma.2017.11.002.

9. Varani J, Perone P, Deming MO, Warner $\mathrm{RL}$, Aslam MN, Bhagavathula N, Dame MK, Voorhees JJ. Impaired keratinocyte function on matrix metalloproteinase-1 (MMP-1) damaged collagen. Arch Dermatol Res. Agto 2009 ; $301(7): 497-506$. Doi: 10.1007/s00403-009-0948-4.

10. Zhen AX, Piao MJ, Kang KA, Fernando PDSM, Kang HK, Koh YS, Hyun JW. Esculetin Prevents the Induction of Matrix Metalloproteinase-1 by Hydrogen Peroxide in Skin Keratinocytes. J Cancer Prev. Jun 2019 ; $24(2): 123-128$. Doi : 10.15430/JCP.2019.24.2.123.

11. Tavelli L, McGuire MK, Zucchelli G, Rasperini G, Feinberg SE, Wang HL, et al. Extracellular matrix-based scaffolding technologies for periodontal and peri-implant soft tissue regeneration. J Periodontol. $2020 ; 91(1): 17-25$. Doi:10.1002/JPER.19-0351

12. Polimeni G, Xiropaidis AV, Wikesjö UM. Biology and principles of periodontal wound healing/regeneration. Periodontol 2000. 2006; 41:30-47. Doi: 10.1111/j.1600-0757.2006.00157.x.

13. Rojas MA, Marini L, Pilloni A, Sahrmann $P$. Early wound healing outcomes after regenerative periodontal surgery with enamel matrix derivatives or guided tissue regeneration: a systematic review. BMC Oral Health. 2019;19(1):76. Doi:10.1186/s12903019-0766-9

14. Laney WR. Glossary of Oral and Maxillofacial Implants. Int J Oral Maxillofac Implants. Jul-agto 2017; 32 ( 4 ) : G i - G 200 . D o i : 10.11607/jomi.2017.4.gomi. 
15. Felzani R. Cicatrización de los tejidos con interés en cirugía bucal: revisión de la literatura. Acta Odontol Venez. 2005: 43(3): 310-8. Disponible en: https://www.actaodontologica.com/edici ones/2005/3/cicatrizacion_tejidos.asp

16. Pellegrini G, Rasperini G, Pagni G, Giannobile WV, Milani S, Musto F, Dellavia C. Local wound healing biomarkers for real-time assessment of periodontal regeneration: pilot study. J Periodontal Res. Jun 2017; 52(3):388396. Doi: $10.1111 /$ jre.12403.

17. Chen YW, Lee CT, Hum L, Chuang SK. Effect of flap design on periodontal healing after impacted third molar extraction: a systematic review and meta-analysis. Int J Oral Maxillofac Surg. Mar 2017; 46(3):363-372. Doi: 10.1016/j.ijom.2016.08.005.

18. Kudiyirickal MG, Pappachan JM. Diabetes mellitus and oral health. Endocrine. May 2015; 49(1):27-34. Doi: 10.1007/s12020-014-0496-3.

19. Kido D, Mizutani K, Takeda K, Mikami R, Matsuura T, Iwasaki K, Izumi Y. Impact of diabetes on gingival wound healing via oxidative stress. PLoS One. 21 Dic 2017; 12(12):e 0189601 . Doi: 10.1371/journal.pone.0189601.

20. Roden M. Diabetes mellitus - Definition, Klassifikation und Diagnose [Diabetes mellitus: definition, classification and diagnosis]. Wien Klin Wochenschr. 2016; 128 Suppl 2:S37-S40. Doi:10.1007/s00508-015-0931-3

21. Chang PC, Tsai SC, Jheng YH, Lin YF, Chen CC. Soft-tissue wound healing by anti-advanced glycation end-products agents. J Dent Res. Apr 2014; $93(4): 388-93$. D o i : $10.1177 / 0022034514523785$.
22. Yamano S, Kuo WP, Sukotjo C. Downregulated gene expression of TGF- $\beta$ s in diabetic oral wound healing. J Craniomaxillofac Surg. Mar 2013; 41 ( 2 ) : e $42-8$. D o i : 10.1016/j.jcms.2012.08.001.

23. Kardeşler L, Buduneli N, Çetinkalp S, Lappin D, Kinane DF. Gingival crevicular fluid IL-6, tPA, PAI-2, albumin levels following initial periodontal treatment in chronic periodontitis patients with or without type 2 diabetes. Inflamm Res. Feb 2011; 60(2):143-51. Doi: 10.1007/s00011-010-0248-7.

24. Mattson JS, Cerutis DR, Parrish LC. Complications associated with diabetes mellitus after guided tissue regeneration--a case report revisited. Compend Contin Educ Dent. Dic 2002; 23(12):1135-8, 1140, 1142 passim; quiz 1146. PMID: 12592715.

25. Ghaisas MM, Kshirsagar SB, Sahane RS. Evaluation of wound healing activity of ferulic acid in diabetic rats. Int Wound J. Oct 2014; 11(5):523-32. Doi: 10.1111/j.1742-481X.2012.01119.x.

26. Li M, Li CZ. High glucose improves healing of periodontal wound by inhibiting proliferation and osteogenetic differentiation of human PDL cells. Int Wound J. Feb 2016; 13(1):39-43. Doi: 10.1111/iwj.12218.

27. Shirakata Y, Eliezer M, Nemcovsky CE, Weinreb $M$, Dard $M$, Sculean $A$, Bosshardt DD, Moses O. Periodontal healing after application of enamel matrix derivative in surgical supra/infrabony periodontal defects in rats with streptozotocin-induced diabetes. J Periodontal Res. Feb 2014; 49(1):93-101. Doi: 10.1111/jre.12084.
Correspondencia:

artdent2000@hotmail.com
Fecha de recepción : 10 de junio de 2020

Fecha de aceptación : 19 de agosto de 2020 